\title{
The effect of gefapixant, a P2X3 antagonist, on cough reflex sensitivity: a randomised placebo-controlled study
}

\author{
Alyn H. Morice ${ }^{1}$, Michael M. Kitt ${ }^{2}$, Anthony P. Ford ${ }^{2}$, Andrew M. Tershakovec ${ }^{2}$, \\ Wen-Chi Wu ${ }^{2}$, Kayleigh Brindle ${ }^{1}$, Rachel Thompson ${ }^{1}$, \\ Susannah Thackray-Nocera ${ }^{1}$ and Caroline Wright ${ }^{1}$ \\ Affiliations: ${ }^{1}$ Hull York Medical School, Cottingham, UK. ${ }^{2}$ Merck \& Co., Inc., Kenilworth, NJ, USA.
}

Correspondence: Alyn H. Morice, Respiratory Medicine, Hull York Medical School, University of Hull, Castle Hill Hospital, Castle Road, Cottingham, East Yorkshire, HU16 5JQ, UK. E-mail: a.h.moricedahull.ac.uk

@ERSpublications

Gefapixant reduces coughing in patients and blocks ATP- and distilled-water-induced cough, but not cough evoked by citric acid or capsaicin, suggesting a unique TRPV4/ATP pathway may underlie cough hypersensitivity seen in chronic refractory cough http://bit.ly/2Gcr9Lr

Cite this article as: Morice $\mathrm{AH}$, Kitt MM, Ford AP, et al. The effect of gefapixant, a P2X3 antagonist, on cough reflex sensitivity: a randomised placebo-controlled study. Eur Respir J 2019; 54: 1900439 [https://doi. org/10.1183/13993003.00439-2019].

ABSTRACT We evaluated the effect of gefapixant on cough reflex sensitivity to evoked tussive challenge.

In this phase 2, double-blind, two-period study, patients with chronic cough (CC) and healthy volunteers (HV) were randomised to single-dose gefapixant $100 \mathrm{mg}$ or placebo in a crossover fashion. Sequential inhalational challenges with ATP, citric acid, capsaicin and distilled water were performed 1,3 and $5 \mathrm{~h}$ after dosing. Mean concentrations evoking $\geqslant 2$ coughs (C2) and $\geqslant 5$ coughs (C5) post dose versus baseline were co-primary endpoints. Objective cough frequency (coughs $\cdot \mathrm{h}^{-1}$ ) over $24 \mathrm{~h}$ and a cough severity visual analogue scale (VAS) were assessed in CC patients. Adverse events were monitored.

$24 \mathrm{CC}$ patients and $12 \mathrm{HV}$ were randomised (mean age 61 and 38 years, respectively). The cough challenge threshold increased for ATP by 4.7 -fold $(\mathrm{C} 2, \mathrm{p} \leqslant 0.001)$ and 3.7 -fold $(\mathrm{C} 5, \mathrm{p}=0.007)$ for gefapixant versus placebo in $\mathrm{CC}$ patients; in $\mathrm{HV}, \mathrm{C} 2$ and $\mathrm{C} 5$ increased 2.4-fold (C2, $\mathrm{p}=0.113$; $\mathrm{C} 5, \mathrm{p}=0.003)$. The distilled water $\mathrm{C} 2$ and $\mathrm{C} 5$ thresholds increased significantly $(\mathrm{p}<0.001)$ by a factor of 1.4 and 1.3 , respectively, in CC patients. Gefapixant had no effect on capsaicin or citric acid challenge. Median cough frequency was reduced by $42 \%$ and the least squares mean cough severity VAS was $18.0 \mathrm{~mm}$ lower for gefapixant versus placebo in CC patients. Dysgeusia was the most frequent adverse event $(75 \%$ of $\mathrm{HV}$ and $67 \%$ of CC patients).

ATP-evoked cough was significantly inhibited by gefapixant $100 \mathrm{mg}$, demonstrating peripheral target engagement. Cough count and severity were reduced in CC patients. Distilled water may also evoke cough through a purinergic pathway.

This article has supplementary material available from erj.ersjournals.com

This study is registered as a clinical trial (NCT02476890). Merck \& Co., Inc.'s data sharing policy, including restrictions, is available at http://engagezone.merck.com/ds_documentation.php Requests for access to the clinical study data can be submitted through the EngageZone site or via email to dataaccess@merck.com

Received: March 042019 | Accepted after revision: April 032019

Copyright OERS 2019 


\section{Introduction}

Chronic cough (CC), i.e. cough lasting $\geqslant 8$ weeks, has been reported in up to $10 \%$ of the general population [1,2]. Patients often experience physical, social and psychological effects from paroxysms of coughing that may be as frequent as hundreds or even thousands of times each day persisting for months or years [3-8].

CC patients may have underlying disorders, including asthma, pulmonary fibrosis, lung cancer, chronic obstructive pulmonary disease (COPD), rhinitis, gastro-oesophageal reflux or oesophageal dysmotility, or they may have unexplained CC for which no associated condition can be identified. In patients with refractory chronic cough (RCC), conventional treatment of underlying disorders is frustratingly inadequate in ameliorating bouts of coughing. Many patients also exhibit an unexplained hypersensitivity to external stimuli, such as a change in temperature, strong smells or aromatic compounds [9]. Cough hypersensitivity syndrome (CHS) is an overarching diagnosis for patients with exquisite sensitivity to otherwise innocuous stimuli [10].

Cough challenge with inhaled tussive agents has been used to assess cough reflex response for several decades [11]. The most common challenge agents include citric acid, capsaicin and fog (i.e. distilled water), which stimulate cough through various peripheral nerve receptors in the airways [12]. ATP also induces cough in conditions such as asthma and COPD [13-15]. Recently, ATP challenge has been characterised in patients with and without CC, and although patients do exhibit a heightened cough reflex, the difference in sensitivity is surprisingly small [16]. Thus, the role of ATP in cough reflex sensitivity remains to be fully elucidated.

P2X3 receptors are ligand-gated ion channels that respond to ATP. Medications targeting these receptors may treat patients through normalisation of afferent sensitivity, specifically afferents that innervate the upper and lower airways [17]. Gefapixant is a P2X3 receptor antagonist that has demonstrated efficacy in the treatment of RCC [18]. To further elucidate the role of purinergic mechanisms in the cough reflex, we conducted a study of gefapixant on cough reflex sensitivity to four inhaled challenge agents, ATP, distilled water, capsaicin and citric acid, in both healthy volunteers (HV) and CC patients. Our hypothesis was that gefapixant would differentially affect cough reflex sensitivity depending on the challenge agent used.

\section{Methods}

This double-blind, randomised, two-period, crossover study (protocol 014; ClinicalTrials.gov NCT02476890) in HV and CC patients was conducted at a single site (Hull York Medical School, Cottingham, UK) in accordance with principles of Good Clinical Practice and was approved by the Yorkshire and the Humber - Sheffield Research Ethics Committee (Jarrow, UK). Subjects provided informed consent prior to being enrolled in the study.

\section{Subjects}

Enrolled HV and CC patients were between 18 and 80 years of age, inclusive, and were non-smokers for at least 5 years. $\mathrm{HV}$ had a forced expiratory volume in $1 \mathrm{~s}\left(\mathrm{FEV}_{1}\right) \geqslant 80 \%$ at screening. CC patients had refractory cough for $\geqslant 1$ year (cough unresponsive to $\geqslant 8$ weeks of treatment for underlying conditions including reflux disease, asthma and rhinitis) and demonstrated significant cough symptoms as determined by a score $>20$ out of 70 on the Hull Airway Reflux Questionnaire (HARQ). Additional exclusion criteria are provided in the supplementary material.

\section{Study design}

After screening, there was a baseline visit before each of two, 1-day treatment periods that were separated by a minimum 48 -h washout period. Treatment consisted of gefapixant $100 \mathrm{mg}$ (two gefapixant 50 -mg tablets) and placebo (two matching placebo tablets). Treatments were administered in a double-blind fashion in which subjects and study personnel were blinded to treatment codes. Subjects were assigned to one of two treatment sequences based on a computer-generated randomisation schedule using a permuted block algorithm to allocate subjects' numbers. Stratification was used (HV versus CC patients). An equal number of subjects was randomly assigned to each sequence.

At baseline and during each treatment period, cough reflex sensitivity was measured by determining the lowest concentration of inhaled solution required to evoke $\geqslant 2$ coughs $(\mathrm{C} 2)$ and the lowest concentration of inhaled solution required to evoke $\geqslant 5$ coughs (C5) for four separate cough challenges (ATP, capsaicin, citric acid and distilled water). The cough challenges were performed in the morning of each baseline visit and 1,3 and $5 \mathrm{~h}$ after dosing during the treatment periods. Objective cough monitoring (from the end of the cough reflex sensitivity challenge to the following day (up to $24 \mathrm{~h}$ ) was performed at baseline and during each of the two treatment periods in CC patients. Subjects returned 2 weeks after their last treatment visit for a follow-up visit. 
The challenge agents were prepared by dilution of stock solutions with saline. The following pre-defined concentrations were used for each challenge agent: ATP: $0.1 \mathrm{mM}, 0.3 \mathrm{mM}, 1 \mathrm{mM}, 3 \mathrm{mM}, 10 \mathrm{mM}, 30 \mathrm{mM}$, $100 \mathrm{mM}, 300 \mathrm{mM}$; capsaicin: $0.3 \mu \mathrm{M}, 1 \mu \mathrm{M}, 3 \mu \mathrm{M}, 10 \mu \mathrm{M}, 30 \mu \mathrm{M}, 100 \mu \mathrm{M}, 300 \mu \mathrm{M}, 1000 \mu \mathrm{M}$; citric acid: $1 \mathrm{mM}, 3 \mathrm{mM}, 10 \mathrm{mM}, 30 \mathrm{mM}, 100 \mathrm{mM}, 300 \mathrm{mM}, 1 \mathrm{M}, 3 \mathrm{M}$; distilled water: $20 \%, 40 \%, 60 \%, 80 \%, 100 \%$ distilled water in $0.9 \%$ saline). Capsaicin and citric acid were obtained from the National Health Services manufacturing pharmacy (Stockport, UK). ATP was obtained from Sigma Aldrich (Gillingham, Dorset, UK).

\section{Primary and secondary endpoints}

The concentration of the challenge agents inducing C2 and C5 were assessed 1, 3 and $5 \mathrm{~h}$ after exposure; for distilled water, the number of coughs generated during $1 \mathrm{~min}$ of exposure was recorded. The co-primary endpoints were the concentrations inducing C2 and C5 for each challenge averaged across the three time points.

Secondary efficacy endpoints included a cough severity visual analogue scale (VAS), an urge-to-cough VAS, cough frequency and total HARQ score in CC patients. CC patients completed the two VAS (100-mm scale) at screening and $1 \mathrm{~h}$ after the final cough challenge during the treatment periods; cough severity was scored from "no cough" to "worst cough"; urge-to-cough was scored from "no urge-to-cough" to "worst urge-to-cough" during the previous $1 \mathrm{~h}$.

An ambulatory recording device was used to measure cough frequency [19]. Change from baseline in objective cough frequency and urge-to-cough were measured during treatment periods 1 and 2 (up to $24 \mathrm{~h}$ for each measure). Recordings were started at the end of the cough challenge protocol and continued overnight until the following day. Each clock hour was compared across the 3 days of recording. A minimum of $5 \mathrm{~h}$ of synchronous and contiguous recording was required before data were considered eligible for analysis.

The HARQ (completed at screening and 1-h post dose during the treatment periods) comprises 14 items, each with a score ranging from "0" (no problem) to "5" (severe/frequent problem) [20]. The total HARQ score is the sum of these 14 item scores with a maximum total score of 70 .

\section{Safety evaluation}

Safety was assessed through monitoring of adverse events (AEs) and serious AEs, physical examinations, vital signs, 12-lead ECGs and clinical laboratory tests (haematology, chemistry and urine analysis).

\section{Statistical methods}

For each challenge, CC patients and HV were analysed separately. C2 and C5 analyses were also performed separately.

Log transformation was used for the co-primary endpoints. A log C2 and C5 concentration was generally regarded as normally distributed within a population, so the treatment comparisons were performed using a mixed effect repeated measures (MMRM) model that included fixed effects for period, treatment group and all interaction terms of treatment, time point and period, with the baseline value (in log scale) as a

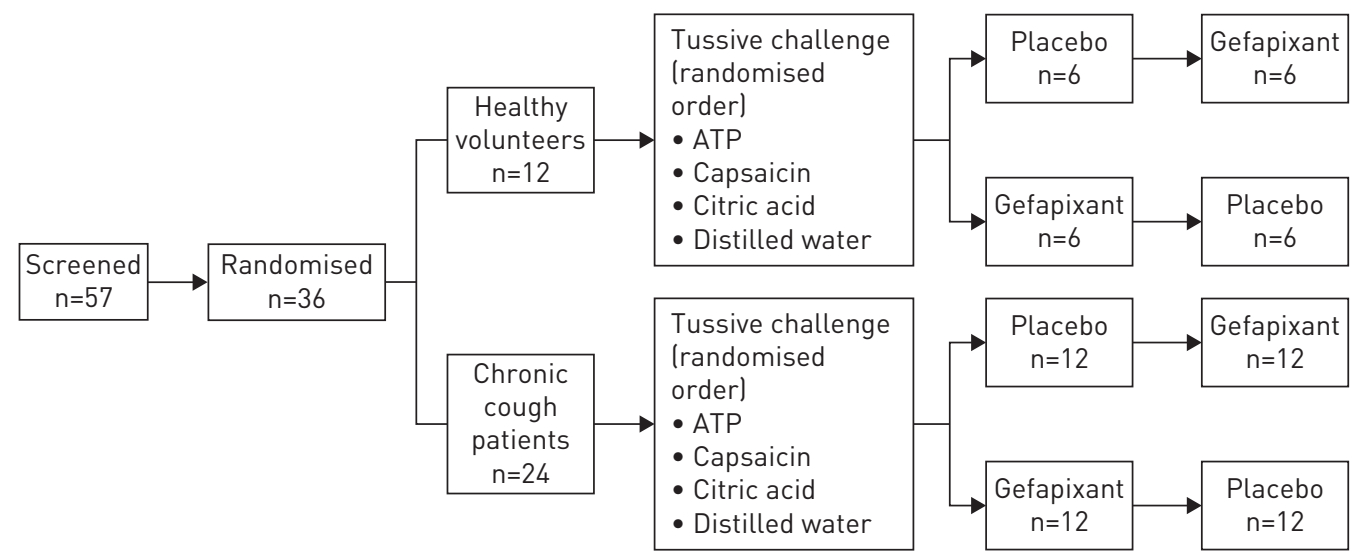

FIGURE 1 Disposition of subjects. Tussive challenges were administered 1,3 and $5 \mathrm{~h}$ post dose. All randomised subjects were included in the full analysis set for efficacy analyses as well as the safety set for evaluation of safety. All randomised subjects completed the study. 
covariate. The MMRM model used all available data at 1, 3 and $5 \mathrm{~h}$ post dose. An unstructured covariance matrix was applied for the MMRM.

For cough reflex sensitivity testing, if a subject did not achieve C2 or C5 at the maximum concentration of the challenge agent, 1.5 times that concentration was imputed.

Secondary endpoints for subjects with CC were analysed using a MMRM model that included fixed effects for period, treatment group and the interaction term of treatment and period, with the period-specific baseline value as a covariate.

\section{TABLE 1 Baseline characteristics}

Healthy volunteers

Chronic cough patients

\begin{tabular}{lcc}
\hline Subjects $\mathbf{n}$ & 12 & 24 \\
Female sex $\mathbf{n}(\%)$ & $11(92)$ & $21(88)$ \\
Age years & $37.8 \pm 8.65$ & $61.1 \pm 8.69$ \\
Age range years & $26-52$ & $48-73$ \\
Weight kg & $71.5 \pm 13.24$ & $69.1 \pm 16.46$ \\
Duration of cough years & $\mathrm{N} / \mathrm{A}$ & $14.6 \pm 9.89$ \\
Duration range years & $\mathrm{N} / \mathrm{A}$ & $3-44$ \\
Cough severity VAS & $\mathrm{N} / \mathrm{A}$ & $68.6 \pm 17.45$
\end{tabular}

Data are presented as mean \pm SD, unless otherwise stated. VAS: visual analogue scale; N/A: not applicable.

TABLE 2 Treatment comparison using a mixed effect repeated measures model for C2 and C5 based on natural log-transformed data for the full analysis set of chronic cough patients and healthy volunteers

C2

C5

\begin{tabular}{|c|c|c|c|c|}
\hline & & \\
\hline & Gefapixant $100 \mathrm{mg}$ & Placebo & Gefapixant $100 \mathrm{mg}$ & Placebo \\
\hline Chronic cough patients $n$ & 24 & 24 & 24 & 24 \\
\hline \multicolumn{5}{|l|}{ ATP mM } \\
\hline Geometric mean & $18.1 *$ & 3.9 & $33.9 * *$ & 9.2 \\
\hline Ratio $(95 \% \mathrm{CI})$ & $4.7(2.0-10.8)$ & & $3.7(1.5-9.2)$ & \\
\hline \multicolumn{5}{|l|}{ Distilled water \% } \\
\hline Geometric mean & $83.4^{* *}$ & 61.8 & $91.0 * *$ & 69.1 \\
\hline Ratio $(95 \% \mathrm{CI})$ & $1.4(1.1-1.6)$ & & $1.3(1.1-1.6)$ & \\
\hline \multicolumn{5}{|l|}{ Capsaicin $\mu \mathrm{M}$} \\
\hline Geometric mean & 5.6 & 4.1 & 10.0 & 7.8 \\
\hline Ratio $(95 \% \mathrm{CI})$ & $1.4(0.8-2.5)$ & & $1.3(0.7-2.4)$ & \\
\hline \multicolumn{5}{|l|}{ Citric acid mM } \\
\hline Geometric mean & 58.6 & 46.5 & 114.6 & 86.5 \\
\hline Ratio $(95 \% \mathrm{Cl})$ & $1.3(0.6-2.6)$ & & $1.3(0.7-2.7)$ & \\
\hline Healthy volunteers $n$ & 12 & 12 & 12 & 12 \\
\hline \multicolumn{5}{|l|}{ ATP $\mathrm{mM}$} \\
\hline Geometric mean & 120.2 & 49.5 & $272.5^{* *}$ & 113.5 \\
\hline Ratio $(95 \% \mathrm{CI})$ & $2.4(0.8-7.4)$ & & $2.4(1.4-4.0)$ & \\
\hline \multicolumn{5}{|l|}{ Distilled water \% } \\
\hline Geometric mean & $111.9 *$ & 76.4 & 127.1 & 100.7 \\
\hline Ratio $(95 \% \mathrm{CI})$ & $1.5(1.3-1.7)$ & & $1.3(0.9-1.8)$ & \\
\hline \multicolumn{5}{|l|}{ Capsaicin $\mu \mathrm{M}$} \\
\hline Geometric mean & 21.1 & 20.8 & 86.8 & 17.7 \\
\hline Ratio $(95 \%$ Cl) & $1.0(0.5-2.0)$ & & $0.8(0.3-1.9)$ & \\
\hline \multicolumn{5}{|l|}{ Citric acid mM } \\
\hline Geometric mean & 475.5 & 272.5 & 1232 & 914.6 \\
\hline Ratio $(95 \% \mathrm{CI})$ & $1.7(0.8-4.0)$ & & $1.4(0.5-3.8)$ & \\
\hline
\end{tabular}

Missing values for the minimum concentration required to induce $\geqslant 2$ coughs (C2) or $\geqslant 5$ coughs (C5) (unable to reach) were imputed using $1.5 \times$ maximum concentration level. The geometric mean was estimated by exponentiating the least squares (LS) mean (in log scale). The ratio of gefapixant to placebo was estimated by exponentiating the LS mean difference (in log scale). *: p-value (LS mean difference versus placebo) $<0.01 ;{ }^{* *}$ : $p$-value (LS mean difference versus placebo) $<0.001$. 


\section{Results}

\section{Subjects}

In total, $24 \mathrm{CC}$ patients and $12 \mathrm{HV}$ were randomised; all subjects completed the study and were included in the primary efficacy population (full analysis set (FAS)) and the safety population (figure 1). Baseline characteristics were comparable between treatment sequences although mean age of $\mathrm{HV}$ was lower (38 years) that that of CC patients (63 years) and more women than men were enrolled. The median duration of CC was 12 years (table 1 ).

\section{Primary endpoints}

Gefapixant was associated with an increase in the concentration of ATP and distilled water required to induce $\mathrm{C} 2$ and $\mathrm{C} 5$ for both HV and CC patients versus placebo.

The ATP cough challenges in CC patients showed a 4.7 -fold $(\mathrm{p}=0.0006)$ concentration increase to induce $\mathrm{C} 2$ and a 3.7-fold ( $\mathrm{p}=0.0067$ ) increase for C5 with gefapixant versus placebo. In HV, a 2.4-fold ( $\mathrm{p}=0.0029)$ increase was seen for C5 (table 2, figure 2); the change at C2 was of a similar degree but did not achieve statistical significance (figure 3). The concentration of distilled water to induce C2 and C5 increased $(\mathrm{p}<0.05)$, but only by a factor of 1.4 to 1.3 in CC patients and 1.5 to 1.3 in HV. Capsaicin and citric acid concentrations did not increase with gefapixant for C2 and C5 in either HV or CC patients (table 2).
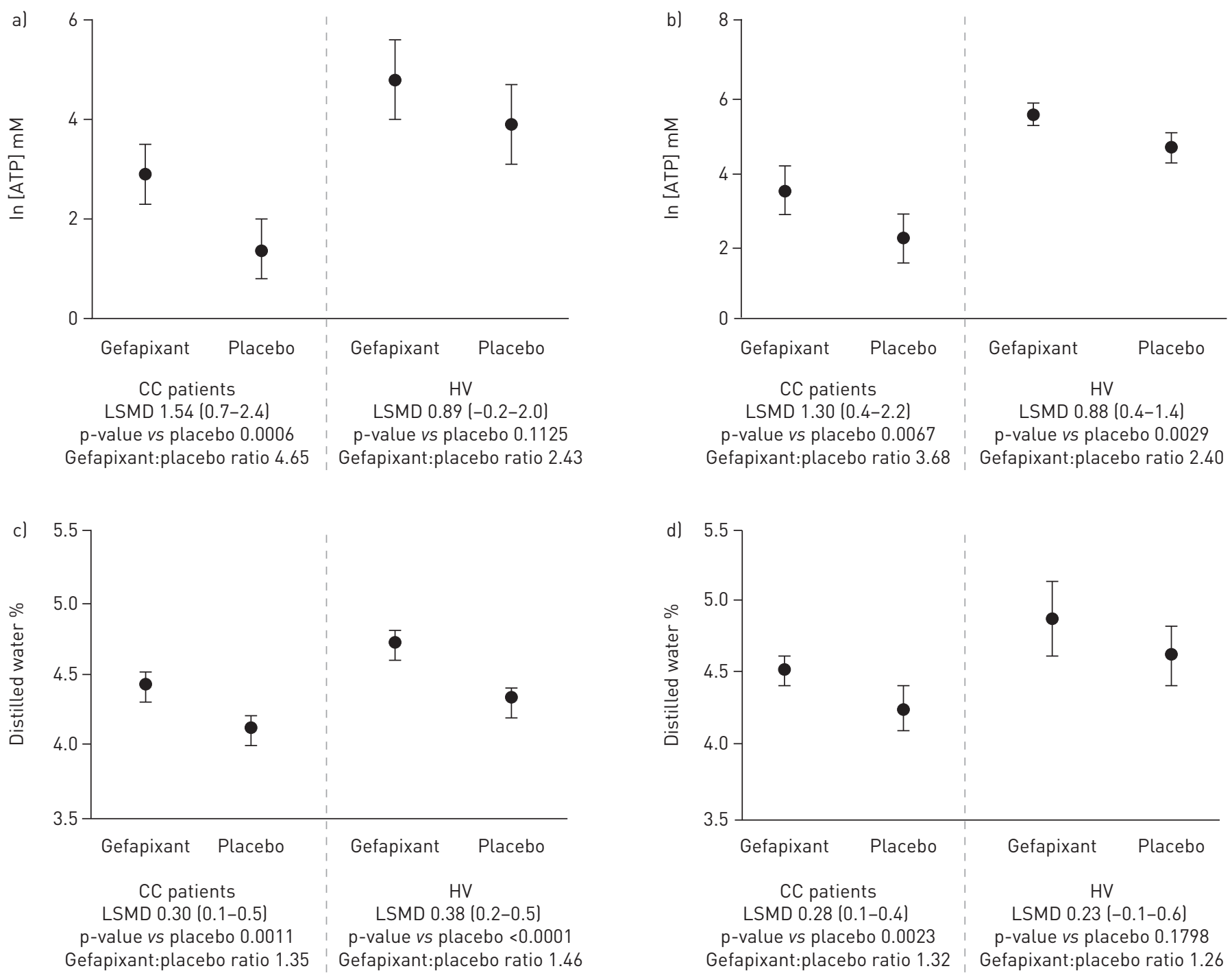

FIGURE 2 Mean concentrations evoking $\geqslant 2$ coughs ( $C 2$ ) and $\geqslant 5$ (C5) coughs for $a, b)$ ATP and $c$, d) distilled water cough challenges. Mixed effect repeated measures analysis based on natural log-transformed data: full analysis set population (primary analysis of the mean post-dose response (hours 1, 3 and 5) versus baseline). Least squares mean difference (LSMD) presented with $95 \%$ confidence intervals. a) C2 mean response to ATP inhalation; b) C5 mean response to ATP inhalation; c) C2 mean response to distilled water; d) C5 mean response to distilled water. CC: chronic cough; HV: healthy volunteers. 

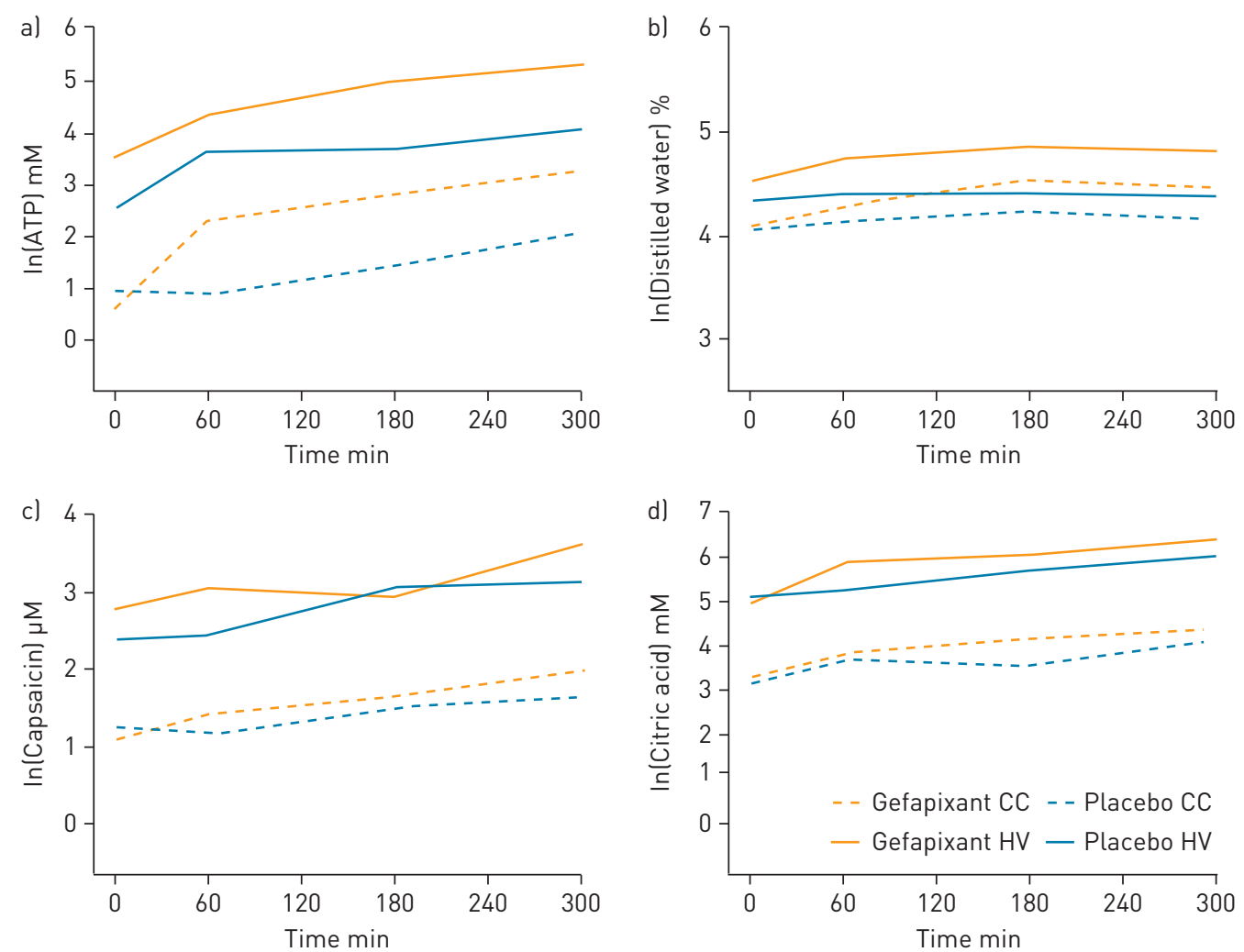

FIGURE 3 Cough challenges. Natural log-transformed mean concentrations evoking $\geqslant 2$ coughs (C2) over time (hours 0, 1, 3 and 5). a) ATP challenge; b) distilled water challenge; c) capsaicin challenge; d) citric acid challenge. CC: chronic cough patients; HV: healthy volunteers.

\section{Secondary endpoints}

Cough severity VAS

A greater reduction in change from baseline on the cough severity VAS was observed with gefapixant versus placebo $(\mathrm{p}=0.004$, table 3$)$.

Urge-to-cough VAS

A greater reduction in change from baseline on the urge-to-cough VAS was observed with gefapixant versus placebo $(\mathrm{p}=0.002$, table 3$)$.

$H A R Q$

A significant reduction from baseline in HARQ total score was observed with gefapixant treatment although a reduction with placebo treatment was also observed and the difference for gefapixant versus placebo did not achieve significance (table 3).

\section{Cough frequency}

A greater reduction from baseline in cough frequency was observed with gefapixant treatment versus placebo $(\mathrm{p}=0.008$, table 3$)$.

\section{Safety}

There was an increased incidence of AEs with gefapixant versus placebo in both HV and CC patients (table 4). No subject had a serious $\mathrm{AE}$ or an $\mathrm{AE}$ leading to discontinuation from the study. The most common AEs were related to taste (i.e. ageusia or dysgeusia) (table 4).

\section{Discussion}

This trial demonstrated that a significant increase in ATP and distilled water concentrations were required to elicit C2 or C5 after dosing with gefapixant $100 \mathrm{mg}$. In contrast, no effect was observed in the capsaicin or citric acid challenges. Responses for all challenges in HV mimicked the responses of CC patients but to a lesser degree. Additionally, gefapixant $100 \mathrm{mg}$ decreased cough severity and frequency among CC patients. 


\begin{tabular}{|c|c|c|c|}
\hline & Subjects $\mathrm{n}$ & LS mean $(95 \% \mathrm{CI})$ & p-value \\
\hline \multicolumn{4}{|l|}{ Cough severity VAS } \\
\hline Gefapixant 100 mg & 24 & $-26.2(-36.2--16.2)$ & \\
\hline Placebo & 24 & $-8.2(-18.7-2.2)$ & \\
\hline Gefapixant versus placebo & & $-18.0(-29.8--6.2)$ & 0.004 \\
\hline \multicolumn{4}{|l|}{ Urge-to-cough VAS } \\
\hline Gefapixant 100 mg & 24 & $-29.8(-38.9--20.7)$ & \\
\hline Placebo & 24 & $-11.7(-20.9--2.6)$ & \\
\hline Gefapixant versus placebo & & $-18(-29.1--7.0)$ & 0.002 \\
\hline \multicolumn{4}{|l|}{ HARQ total score } \\
\hline Gefapixant $100 \mathrm{mg}$ & 24 & $-16.2(-22.1--10.3)$ & \\
\hline Placebo & 24 & $-11.0(-17.0--5.1)$ & \\
\hline Gefapixant versus placebo & & $-5.2(-10.9-0.6)$ & 0.077 \\
\hline \multicolumn{4}{|c|}{ Cough frequency over $24 \mathrm{~h}$ coughs $\cdot \mathrm{h}^{-1}$} \\
\hline Gefapixant $100 \mathrm{mg}$ & 24 & $-7.7(-10.1--5.3)$ & \\
\hline Placebo & 22 & $-4.1(-6.5--1.7)$ & \\
\hline Gefapixant versus placebo & & $-3.6(-6.2--1.0)$ & 0.008 \\
\hline
\end{tabular}

\begin{tabular}{|c|c|c|c|c|}
\hline & \multicolumn{2}{|c|}{ Healthy volunteers } & \multicolumn{2}{|c|}{ Chronic cough patients } \\
\hline & $\begin{array}{l}\text { Gefapixant } \\
100 \mathrm{mg}\end{array}$ & Placebo & $\begin{array}{l}\text { Gefapixant } \\
100 \mathrm{mg}\end{array}$ & Placebo \\
\hline Subjects $\mathrm{n}$ & 12 & 12 & 24 & 24 \\
\hline Any AEs & $12(100.0)$ & $6(50.0)$ & 23 (95.8) & 8 (33.3) \\
\hline $\begin{array}{l}\text { Serious AEs or AEs leading to } \\
\text { discontinuation }\end{array}$ & 0 & 0 & 0 & 0 \\
\hline \multicolumn{5}{|l|}{ Most frequent AEs } \\
\hline Dysgeusia & 9 (75.0) & $1(8.3)$ & $16(66.7)$ & 0 \\
\hline Ageusia & $6(50.0)$ & $1(8.3)$ & $7(29.2)$ & 0 \\
\hline Dry mouth & 4 (33.3) & 0 & $6(25.0)$ & $1(4.2)$ \\
\hline Hypoaesthesia (oral) & $3(25.0)$ & 0 & $4(16.7)$ & 0 \\
\hline Headache & 0 & $3(25.0)$ & $6(25.0)$ & 2 (8.3) \\
\hline Paraesthesia (oral) & 1 (8.3) & $1(8.3)$ & $4(16.7)$ & 2 (8.3) \\
\hline
\end{tabular}

Data are presented as $\mathrm{n}(\%)$, unless otherwise stated. AE: adverse event.

The primary function of the cough reflex is to prevent or minimise aspiration. People with conditions in which cough reflex sensitivity is diminished (e.g. stroke, parkinsonism or dementia) frequently experience such events $[21,22]$. It is unsurprising, then, that a series of nociceptors located in the upper airways, attached to vagal afferents, have evolved to defend the airway against such insults. The investigation of cough reflex sensitivity by inhalational tussive challenge has been used for over 60 years as a tool to study the physiology and clinical pharmacology of this vital protective reflex. Citric acid was the first agent to be used and, although its precise mechanism of action is still unclear, this challenge is related to the buffered $\mathrm{pH}$ of the solution used [23]. Capsaicin acts through a specific nociceptor, transient receptor potential cation channel subfamily $\mathrm{v}$ member 1 (TRPV1), which is also acid sensitive but has different characteristics of adaptation [24]; evoked cough can be blocked by specific TRPV1 antagonists [25]. Distilled water again has different attributes, with very rapid adaptation and marked tachyphylaxis. It is thought to trigger cough via osmoreceptors. Finally, the most recently described tussive challenge agent, ATP, produces a concentration-dependent increase in coughing with a slightly greater response seen in those with CC [16]. This latter phenomenon of increased sensitivity to challenge agents is seen with all modalities of cough challenge, but the effect size is small, implying that increased peripheral nociceptor sensitivity may not be a fundamental mechanism in the profound hypersensitivity seen in CHS [26]. 
These challenge agents are thought to act in the immediate vicinity of the airway epithelium. Buffering will rapidly occur with the small droplets of distilled water fog and citric acid. ATP is rapidly metabolised to AMP and adenosine. Capsaicin is highly lipid soluble and avidly taken up into cell membranes. The more central pathways of the vagal afferents through the nodose and jugular ganglia to the solitary nucleus are extremely complex and varied, and exhibit marked plasticity and redundancy in disease [27]. In this environment, the interpretation of cough challenge studies must be undertaken with care.

Our finding that gefapixant led to increases in concentrations needed to induce multiple coughs upon ATP exposure is consistent with peripheral target engagement of the ATP-activated P2X3 receptors in the pathophysiology of CC [28]. It suggests that release of ATP by airway cells may directly stimulate afferent nerves, causing coughing. However, the rapid metabolism of ATP would imply continuous release of ATP to stimulate $\mathrm{P} 2 \mathrm{X} 3$, a receptor with a purportedly rapid desensitisation [29]; this observation is compatible with the brief coughing bouts seen following ATP inhalation. A notable other finding in our study was the significant, although smaller, effect of gefapixant in the distilled water challenge. In a recent paper, Bonvini et al. [30] describe a mechanism whereby hypo-osmolarity could lead to ATP release. TRPV4 is a nociceptor widely located in the airways and activated by hypo-osmotic stimuli [31]. The authors show that activation of TRPV4 causes a release of ATP via pannexin channels and subsequent ATP activation of the neuron can be blocked by a P2X3 antagonist. Administration of a TRPV4 agonist produced prolonged firing of both guinea pig and human A $\delta$ vagus nerve fibres. The antagonist had no effect on citric acid- or capsaicin-sensitive $\mathrm{C}$ fibres. As in the current study, P2X3 antagonism also had no effect on the cough sensitivity of guinea pigs to capsaicin challenge.

These findings suggest that there are at least two distinct pathways engendering the cough reflex: one, the TRPV4/ATP pathway responsible for cough hypersensitivity, and two, direct stimulation of nociceptors. Inhibition of TRPV1 and transient receptor potential cation channel subfamily a member 1 (TRPA1) by specific antagonists has no effect in CC [25, 32], whereas, as we show here, even a single dose of gefapixant inhibits ATP receptors to produce a significant improvement. We believe that this is the first demonstration in humans of two separate sensory pathways evoking cough, with TRPV4/ATP as the most likely candidate mechanism underlying cough hypersensitivity. However CC is most likely a heterogeneous phenomenon, triggered by a variety of peripheral mechanisms, thus explaining the significant subgroup of non-responders to gefapixant seen in phase 2 studies. Presumably cough in these patients is mediated via other, non-P2X2/3-related mechanisms.

Gefapixant has been evaluated in patients with CC at doses ranging from $7.5 \mathrm{mg}$ to $600 \mathrm{mg}$ twice daily $[18,33-35]$. A proof-of-concept study demonstrated efficacy at the high dose of $600 \mathrm{mg}$ twice daily [18] and subsequent dose-ranging studies demonstrated efficacy in doses from $15 \mathrm{mg}$ to $50 \mathrm{mg}$ twice daily with no apparent efficacy advantage with doses above $50 \mathrm{mg}$ twice daily [33-35]. In this study, a single dose of gefapixant $100 \mathrm{mg}$ demonstrated a significant reduction in objective cough frequency and positive improvements in patient-reported outcomes on cough severity, urge-to-cough and improved quality of life in CC patients. These effects after a single 100-mg dose are notable because patient-reported outcomes are often delayed in onset when compared with objective scores in studies of CC; these findings confirm the very rapid onset of action seen with a P2X3 receptor antagonist.

Results observed with ATP and, to a lesser extent, distilled water demonstrate their possible utility for assessing agents that target purinergic receptors such as P2X3, although their use as diagnostic tools for CHS appears to be limited. A previous study showed that although CC patients had significantly more coughing at lower concentrations of ATP, they did not appear to have an intrinsically heightened sensitivity to ATP [16]. Perhaps this indicates that although ATP may constitute the final common mediator for cough hypersensitivity, it may not be the excitatory cause of neural sensitisation. Our putative surrogate for TRPV4 activation, distilled water, had an even lesser response to P2X3 antagonism and has a greater degree of adaptation than ATP. Both agents were administered directly to the airways and it may be that the seat of pathological hypersensitivity is located more centrally.

Gefapixant was associated with taste disturbance AEs at the dose of $100 \mathrm{mg}$ in this study. Although gefapixant has generally not been associated with serious AEs, taste disturbances are the most commonly reported AEs $[18,33]$. Previous preclinical research has identified that P2X receptors, particularly P2X2/3 receptors, play an important role in the transmission of taste signals [36, 37]. Studies of purinergic P2X2/3 double-genetic knockout mice have demonstrated a loss of taste-evoked activity [38]. Previous studies with gefapixant suggest a mechanistic role in taste disturbance from P2X3 antagonism based on dose-related taste disturbance [33-35]. Effects on cough reduction were observed at lower doses for which taste disturbances were more limited or minimal; phase 3 studies are ongoing and will provide further evidence of whether positive improvements in the treatment of CC can be achieved with acceptable safety and tolerability [33-35].

There are several important limitations to this study. Taste disturbance may well have influenced the results by unblinding participants. A single dose may not represent effects that occur with chronic therapy. 
A further important limitation for this study is its small sample size, which limited our ability to assess an impact from the testing order of tussive agents. However, for individual subjects, the order remained the same for each study day and randomisation was carried out between patients in a block design to minimise the risk of any order effect, which would be balanced by the crossover nature of the study. Previous studies have demonstrated significant cross tachyphylaxis between challenges and a tendency for a reduced response on repeated challenge. This latter phenomenon may account for the upward drift of the cough challenges with time seen in figure 3. However, a post hoc analysis of the effect of challenge order in this study found no evidence of carryover between different challenges [39].

In summary, we demonstrated that purine ATP-evoked cough was inhibited by gefapixant $100 \mathrm{mg}$ in both $\mathrm{HV}$ and CC patients, although results were more limited in HV. To a smaller, but statistically significant degree, cough was also reduced following the distilled water challenge. An effect of gefapixant on capsaicin- or citric-acid-evoked cough for either HV or CC patients was not observed. Knowledge of the mechanism of drug action is required to understand the relevance of challenge agents in an antitussive drug-discovery model. In this experimental design we have been able to differentiate at least two separate pathways for evoked cough challenge in humans, with the TRPV4/ATP axis most likely to underlie cough hypersensitivity.

Acknowledgements: The authors thank Susan Lu (Merck \& Co., Inc., Kenilworth, NJ, USA) for contributions to the interpretation of the study and scientific review of the manuscript. Anish Mehta (Merck \& Co., Inc., Kenilworth, NJ, USA) provided writing and editorial support for the manuscript. Jennifer Pawlowski (Merck \& Co., Inc., Kenilworth, NJ, USA) assisted with editorial and administrative support.

Author contributions: A.H. Morice: conception/design/planning of the study, acquisition of data, analysis of data, interpretation of results, drafting the manuscript and critical review/revision of the manuscript; M.M. Kitt: conception/ design/planning of the study, acquisition of data, analysis of data, interpretation of results and critical review/revision of the manuscript; A.P. Ford: conception/design/planning of the study, interpretation of results and critical review/revision of the manuscript; A.M. Tershakovec: interpretation of results and critical review/revision of the manuscript; W-C. Wu: analysis of data and critical review/revision of the manuscript; K. Brindle: acquisition of data and critical review/revision of the manuscript; R. Thompson: acquisition of data and critical review/revision of the manuscript; $S$. Thackray-Nocera: acquisition of data and critical review/revision of the manuscript; C. Wright: acquisition of data and critical review/ revision of the manuscript.

Support statement: This study was funded by Merck Sharp \& Dohme Corp., a subsidiary of Merck \& Co., Inc., Kenilworth, NJ, USA (the study was originally conducted and funded by Afferent Pharmaceuticals, which was acquired by Merck \& Co., Inc., Kenilworth, NJ, USA). Funding information for this article has been deposited with the Crossref Funder Registry.

Conflict of interest: A.H. Morice has received consulting fees from Afferent, Merck Sharp \& Dohme, Boehringer Ingelheim, Pfizer, and Proctor \& Gamble; lecture fees from Boehringer Ingelheim and AstraZeneca; and grant support from Proctor \& Gamble. M.M. Kitt is a former employee of Afferent Pharmaceuticals and Merck Sharp \& Dohme Corp., a subsidiary of Merck \& Co., Inc., Kenilworth, NJ, USA, and may own stock in the company. A.P. Ford is a former employee of Afferent Pharmaceuticals and Merck Sharp \& Dohme Corp., a subsidiary of Merck \& Co., Inc., Kenilworth, NJ, USA, and may own stock in the company. A.M. Tershakovec is a former employee of Merck Sharp \& Dohme Corp., a subsidiary of Merck \& Co., Inc., Kenilworth, NJ, USA, and may own stock in the company. W-C. Wu was an employee of Merck Sharp \& Dohme, Corp, during the conduct of the study. K. Brindle has nothing to disclose. R. Thompson has nothing to disclose. S. Thackray-Nocera has nothing to disclose. C. Wright has nothing to disclose.

\section{References}

1 Song WJ, Chang YS, Faruqi S, et al. The global epidemiology of chronic cough in adults: a systematic review and meta-analysis. Eur Respir J 2015; 45: 1479-1481.

2 Morice AH, Fontana GA, Sovijarvi AR, et al. The diagnosis and management of chronic cough. Eur Respir J 2004; 24: 481-492.

3 Everett CF, Kastelik JA, Thompson RH, et al. Chronic persistent cough in the community: a questionnaire survey. Cough 2007; 3: 5.

4 French CL, Crawford SL, Bova C, et al. Change in psychological, physiological, and situational factors in adults after treatment of chronic cough. Chest 2017; 152: 547-562.

5 Kuzniar TJ, Morgenthaler TI, Afessa B, et al. Chronic cough from the patient's perspective. Mayo Clin Proc 2007; 82: 56-60.

6 Chamberlain SA, Garrod R, Douiri A, et al. The impact of chronic cough: a cross-sectional European survey. Lung 2015; 193: 401-408.

7 Dicpinigaitis PV, Tso R, Banauch G. Prevalence of depressive symptoms among patients with chronic cough. Chest 2006; 130: 1839-1843.

8 French CL, Irwin RS, Curley FJ, et al. Impact of chronic cough on quality of life. Arch Intern Med 1998; 158: $1657-1661$.

9 Johansson A, Millqvist E, Nordin S, et al. Relationship between self-reported odor intolerance and sensitivity to inhaled capsaicin: proposed definition of airway sensory hyperreactivity and estimation of its prevalence. Chest 2006; 129: 1623-1628.

10 Morice AH, Millqvist E, Belvisi MG, et al. Expert opinion on the cough hypersensitivity syndrome in respiratory medicine. Eur Respir J 2014; 44: 1132-1148. 
11 Bickerman HA, Barach AL. The experimental production of cough in human subjects induced by citric acid aerosols; preliminary studies on the evaluation of antitussive agents. Am J Med Sci 1954; 228: 156-163.

12 Morice AH, Kastelik JA, Thompson R. Cough challenge in the assessment of cough reflex. Br J Clin Pharmacol 2001; 52: 365-375.

13 Basoglu OK, Barnes PJ, Kharitonov SA, et al. Effects of aerosolized adenosine 5'triphosphate in smokers and patients with COPD. Chest 2015; 148: 430-435.

14 Basoglu OK, Pelleg A, Essilfie-Quaye S, et al. Effects of aerosolized adenosine 5'-triphosphate versus adenosine 5 -monophosphate on dyspnea and airway caliber in healthy nonsmokers and patients with asthma. Chest 2005; 128: 1905-1909.

15 Pellegrino R, Wilson O, Jenouri G, et al. Lung mechanics during induced bronchoconstriction. J Appl Physiol 1996; 81: 964-975.

16 Fowles HE, Rowland T, Wright C, et al. Tussive challenge with ATP and AMP: does it reveal cough hypersensitivity? Eur Respir J 2017; 49: 1601452.

17 Khakh BS, North RA. P2X receptors as cell-surface ATP sensors in health and disease. Nature 2006; 442: 527-532.

18 Abdulqawi R, Dockry R, Holt $\mathrm{K}$, et al. P2X3 receptor antagonist (AF-219) in refractory chronic cough: a randomised, double-blind, placebo-controlled phase 2 study. Lancet 2015; 385: 1198-1205.

19 Barry SJ, Dane AD, Morice AH, et al. The automatic recognition and counting of cough. Cough 2006; 2 : 8.

20 Morice AH, Faruqi S, Wright CE, et al. Cough hypersensitivity syndrome: a distinct clinical entity. Lung 2011; 189: 73-79.

21 Ebihara S, Saito H, Kanda A, et al. Impaired efficacy of cough in patients with Parkinson disease. Chest 2003; 124: 1009-1015.

22 Ebihara S, Sekiya H, Miyagi M, et al. Dysphagia, dystussia, and aspiration pneumonia in elderly people. $J$ Thorac Dis 2016; 8: 632-639.

23 Wong CH, Matai R, Morice AH. Cough induced by low pH. Respir Med 1999; 93: 58-61.

24 Morice AH, Higgins KS, Yeo WW. Adaptation of cough reflex with different types of stimulation. Eur Respir $J$ 1992; 5: 841-847.

25 Belvisi MG, Birrell MA, Wortley MA, et al. XEN-D0501, a novel transient receptor potential Vanilloid 1 antagonist, does not reduce cough in patients with refractory cough. Am J Respir Crit Care Med 2017; 196: 1255-1263.

26 Rai ZL, Fowles HE, Wright $\mathrm{C}$, et al. The effect of $\mathrm{pH}$ on citric acid cough challenge: a randomised control trial in chronic cough and healthy volunteers. Respir Physiol Neurobiol 2018; 257: 51-54.

27 Mazzone SB, Undem BJ. Vagal afferent innervation of the airways in health and disease. Physiol Rev 2016; 96: 975-1024.

28 Ford AP, Undem BJ. The therapeutic promise of ATP antagonism at P2X3 receptors in respiratory and urological disorders. Front Cell Neurosci 2013; 7: 267.

29 Kowalski M, Hausmann R, Schmid J, et al. Flexible subunit stoichiometry of functional human P2X2/3 heteromeric receptors. Neuropharmacology 2015; 99: 115-130.

30 Bonvini SJ, Birrell MA, Grace MS, et al. Transient receptor potential cation channel, subfamily V, member 4 and airway sensory afferent activation: role of adenosine triphosphate. J Allergy Clin Immunol 2016; 138: 249-261.

31 Jia Y, Wang X, Varty L, et al. Functional TRPV4 channels are expressed in human airway smooth muscle cells. Am J Physiol Lung Cell Mol Physiol 2004; 287: L272-L278.

32 Morice AH. TRPA1 receptors in chronic cough. Pulm Pharmacol Ther 2017; 47: 42-44.

33 Smith JA, Kitt MM, Morice AH, et al. MK-7264, a P2X3 receptor antagonist, reduces cough frequency in patients with refractory chronic cough: results from a randomized, controlled, phase $2 \mathrm{~b}$ clinical trial. Am J Respir Crit Care Med 2017; 195: A7608.

34 Smith JA, Kitt M, Sher M, et al. A phase 2 dose-escalation study with AF-219, a P2X3 antagonist for the treatment of chronic cough. Am J Respir Crit Care Med 2016; 193: A6524.

35 Smith J, Kitt M, Sher M, et al. Tackling the burden of chronic cough: a dose escalation study of AF-219. Eur Respir J 2016; 48: Suppl. 60, OA1976.

36 Bo X, Alavi A, Xiang Z, et al. Localization of ATP-gated P2X2 and P2X3 receptor immunoreactive nerves in rat taste buds. Neuroreport 1999; 10: 1107-1111.

37 Finger TE, Danilova V, Barrows J, et al. ATP signaling is crucial for communication from taste buds to gustatory nerves. Science 2005; 310: 1495-1499.

38 Eddy MC, Eschle BK, Barrows J, et al. Double P2X2/P2X3 purinergic receptor knockout mice do not taste NaCl or the artificial sweetener SC45647. Chem Senses 2009; 34: 789-797.

39 James N, Cheung W, Wright CE, et al. Tachyphylaxis and cough reflex sensitivity. Eur Respir J 2018; 52: Suppl. 62, PA4438. 